\title{
Flexibility of Dicer Studied by Implicit Solvent Molecular Dynamics Simulations
}

\author{
J. Sarzyńska ${ }^{1 *}$, A. Mickiewicz ${ }^{1}$, M. Milostan ${ }^{2}$, P. Lukasiak ${ }^{1,2}$, \\ J. Błażewicz ${ }^{1,2}$, M. Figlerowicz ${ }^{1}$ T. Kuliński ${ }^{1}{ }^{*}$ \\ ${ }^{1}$ Institute of Bioorganic Chemistry, Polish Academy of Sciences \\ ul. Noskowskiego 12/14, 61-704 Poznań, Poland \\ *e-mail: \{joanna.sarzynska/tadeusz.kulinski\}@ibch.poznan.pl \\ ${ }^{2}$ Institute of Computing Sciences, Poznan University of Techonology \\ ul. Piotrowo 2, 60-965 Poznań, Poland
}

(Received: 14 December 2009; revised: 2 March 2010; accepted: 10 March 2010; published online: 19 May 2010)

\begin{abstract}
Dicer is an enzyme responsible for processing double-stranded RNAs and plays a key role in an RNAi mechanism. Structural insight into the Dicer is provided by the crystal structure of eukaryotic Dicer from Giardia intestinalis. It has been proposed that the structure has three structurally rigid regions that are connected by the flexible hinges. Flexibility of the Dicer is believed to be a critical feature for its function. Spatial arrangement of the RNA-recognition and the catalytic regions is crucial for producing small RNAs of defined length. It has been suggested that in the Giardia Dicer a Platform domain may help in specific arrangement of these regions. To learn more about the role of the Platform domain in Giardia Dicer, we have performed molecular dynamics (MD) simulations of the whole Dicer (WT Dicer) and the Dicer with a deleted platform domain ( $\Delta$ Plf Dicer). The MD simulations were carried out in an implicit solvent model with two implementations of analytic Generalized Born (GB) solvation model in CHARMM: GBMV (Generalized Born using Molecular Volume) and GBSW (Generalized Born with simple Switching). To detect the key global motions of the Dicer, a principal component analysis (PCA) of the obtained MD trajectories has been used. To further explore the motion of the Dicer, we performed a domain motion analysis with the DYNDOM program. The simulations show that both WT Dicer and $\Delta$ Plf Dicer display flexibility which can be described as a movement of two or three domains. The removal of the Platform substantially changed the flexibility and arrangement of these domains. During the MD simulations of $\Delta$ Plf Dicer an large movement of the RNA-recognition domain was observed.
\end{abstract}

Keywords: implicit solvent model, principal component analysis, molecular dynamics, domain motions, Giardia Dicer

\section{INTRODUCTION}

Dicer is a ribonuclease III (RNase III) family enzyme that cleaves double stranded RNA precursors, producing short interfering RNAs (siRNA) and microRNAs (miRNA). Dicer products are typically $21-25$ nucleotide long, have a monophosphate group at the 5' ends and 3'-dinucleotide overhangs. These small RNAs generated by Dicer initiate a mechanism of gene silencing known as RNA interference.

Structural insight into the Dicer comes from the crystal structure of eukaryotic Dicer from Giardia intestinalis [1] (Fig. 1a). The enzyme contains a PAZ domain and two RNase domains (RNase IIIa and RNase IIIb) that form an internal heterodimer. The PAZ domain is connected with the RNase IIIa by a long $\alpha$ helix (connector helix). The connector helix is additionally supported by N-terminal residues that form a Platform domain. The PAZ domain contains the RNA binding pocket that is recognized by 3' two-base overhang. The crystal structure of the Giardia Dicer reveals that the length of small RNAs produced by Dicer is determined by the distance between the PAZ domain and the catalytic sites in RNase IIIa domain. Biochemical studies have showed that the Dicer enzyme without the PAZ domain produces the RNA of a variable size [2].

The comparison of four copies of the Dicer molecule from the crystallographic asymmetric unit of the Giardia Dicer and the normal mode analysis (NMA) indicate on the flexibility of the molecule [3]. It has been proposed that the structure has three structurally rigid regions that are connected by the flexible hinges. Flexibility of the Dicer is believed to be a critical feature for its function.

Most of the higher eukaryotic Dicers, including human Dicer and dicer-like proteins from Arabidopsis, typically contain also helicase domain and small domain of unknown 
function (DUF283) at the N-terminus and one or two dsRNA-binding domains (dsRBD) at the C-terminus. Yet it is not clear if the Dicers of higher eukaryotes contain the Platform domain present in Giardia Dicer. It has been suggested that the DUF283 domain can form a structure similar to the Platform [1]; however, another authors proposed that DUF283 domain had a double-stranded RNA-binding fold [4]. Also the role of the platform domain in Giardia Dicer is not fully understood. It has been shown that the positively charged platform residues participate in the biding of dsRNA [2]. Location of this domain between the dsRNA binding site and the catalytic center could suggest that it may help in maintaining the mutual arrangement of PAZ and RNase domains.

To learn more about the role of the Platform domain in Dicer from Giardia, we have performed molecular dynamics (MD) simulations in implicit solvent of the whole Dicer (WT Dicer) and the Dicer with a deleted platform domain $(\Delta$ Plf Dicer) (Fig. 1). In this work we have compared the conformational flexibility of WT Dicer and $\Delta$ Plf Dicer.
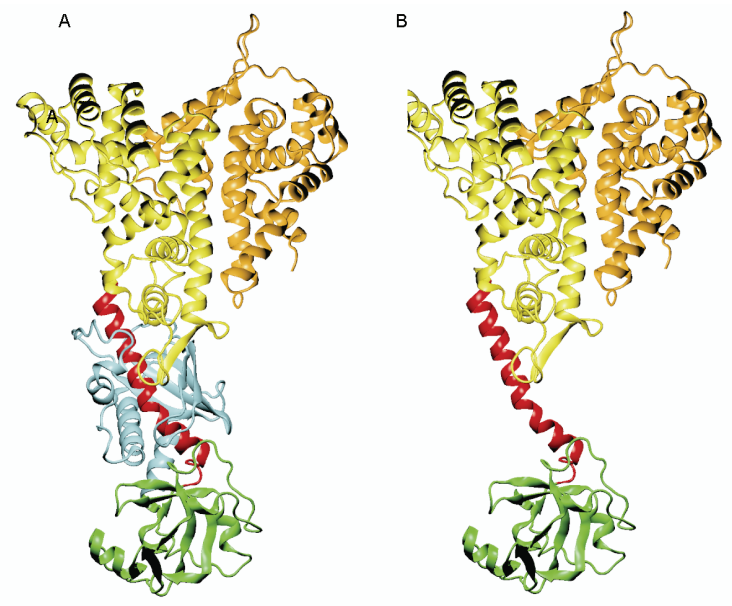

Fig. 1. Models used for MD simulations. A) Crystal structure of wild-type Giardia intestinalis Dicer (PDB code 2FFL), the N-terminal Platform domain (cyan), the PAZ domain (green), the connector helix (red), the RNase region comprising RNase IIIa (yellow) and RNase IIIb (orange). B) Model of $\Delta$ Plf Dicer (deleted Platform domain)

For realistic modeling of biomolecules it is necessary to take into account the solvent environment. The most accurate theoretical approach uses for MD simulations explicit solvent molecules placed around the molecule of interest. However, for large biomolecules explicit inclusion of the water molecules significantly increases the system size and thus the associated computational cost. An alternative is to perform simulations in implicit solvent model that substantially reduces the system size, and improve the conformational sampling due to reduction of the solvent viscosity. Many successful applications of the implicit solvent methods for study of biomolecules, including large scale motion of protein and protein-nucleic acids complexes have been reported [5-7].

Popular implicit solvent models approximate the solvent as a dielectric continuum. Total potential energy of the system is calculated as

$$
E_{\text {tot }}=E_{\mathrm{MM}}+\Delta G_{\text {solv }}
$$

where $E_{\mathrm{MM}}$ represents molecular mechanics potential energy in vacuum and $\Delta \mathrm{G}_{\text {solv }}$ represents the solvation free energy. Within implicit solvent framework $\Delta G_{\text {solv }}$ is estimate as

$$
\Delta G_{\text {solv }}=\Delta G_{\mathrm{el}}+\Delta G_{\text {nonpolar }} .
$$

The electrostatic contribution to the total solvation free energy $\left(\Delta \mathrm{G}_{\mathrm{el}}\right)$ can be calculated directly by solving the Poisson-Boltzman (PB) equation or approximate by a generalized Born (GB) theory. The GB method is preferred for MD simulations due to its higher computational efficiency and similar level of accuracy as PB.

Here we have compared the results of MD simulations obtained with two implementations of GB method in CHARMM: GBMV (Generalized Born using Molecular Volume) $[8,9]$ and GBSW (Generalized Born with simple Switching) $[10,11]$. The GBMV and GBSW methods differ in the way of calculation a dielectric boundary. In the GBMV method the molecular surface is calculated whereas in GBSW the Van der Waals smoothed surface.

\section{METHODS}

\section{II.1. MD simulations}

The initial structure for wild-type Dicer was derived from the crystal structure (PDB code 2FFL, chain C). Missing residues in the crystal structure were completed using MODELLER program [12]. A truncated model of Dicer $(\Delta \mathrm{Plf})$ was obtained by deleting N-terminal 135-residue Platform domain from wild-type Dicer. Simulations were carried out using the CHARMM program $[13,14]$, version $34 \mathrm{~b} 2$ and Charmm22/CMAP force field $[15,16]$. Simulations were performed in a continuum solvent described by a generalized Born model, based on GBMV and GBSW methods as implemented in CHARMM. Default GBMV and GBSW parameters were used. Force switching was applied to switching off the nonbonding and electrostatic solvation contributions to zero from 16 to $18 \AA$. For the GBSW method we used the optimized Born radii for proteins [17], and for the GBMV 
method the Van der Waals radii. Initial structures were relaxed by energy minimization with gradual reducing harmonic restrains on the backbone heavy atoms. Then the protein was subjected to $50 \mathrm{ps}$ of MD and at $100 \mathrm{~K}$ and then at $200 \mathrm{~K}$. After that the MD simulations was continued at $300 \mathrm{~K}$. In all cases the Langevin dynamics with the friction coefficient $10 \mathrm{ps}^{-1}$ for all nonhydrogen atoms and the leapfrog Verlet integrator algorithm was carried out. We used time step of 1.5 fs for GBMV method and 2 fs for GBSW method. The bond lengths involving hydrogen atoms were constrained by the SHAKE method [18].

\section{II.2. Analysis of MD trajectories}

\section{PCA analysis}

The PCA analysis was performed for the entire trajectories with the GROMACS package, version 4.0 [19]. The results were visualized using the Interactive Essential Dynamics (IED) graphical interface [20] within Visual Molecular Dynamics (VMD) [21]. The "porcupine" plots that visualize the eigenvectors were made using perl script within the CCPB software [22].

\section{Domain motion analysis}

The DYNDOM program [23, 24] with default parameters was used to determine dynamic domains, interdomain hinge-bending regions and hinge axes. For the DYNDOM analysis we used two extreme structures obtained from the projection of the trajectory into the first eigenvector.

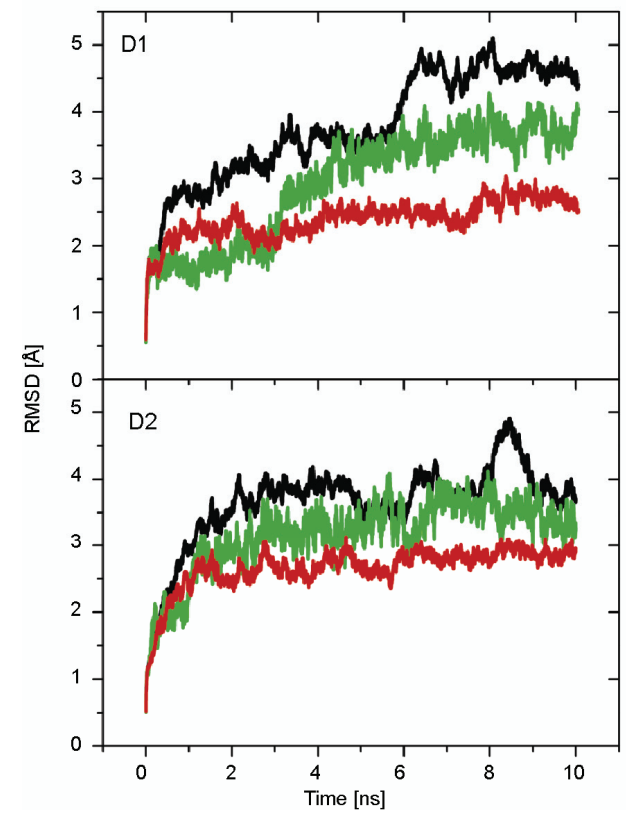

\section{RESULTS}

\section{III.1. Overall stability}

We have performed MD simulations of WT Dicer and $\triangle$ Plf Dicer with GB implicit solvent employing the GBMV and GBSW methods (Table 1). As illustrated on Fig. 2, in both simulations of WT Dicer (D1 and D2 runs) the RNase regions remained stable. For D1 run the RMSD for the overall structure, after a rise at $6 \mathrm{~ns}$, established at $4.6 \pm 0.2 \AA$. For the PAZ domain a rise of the RMSD was observed at $3 \mathrm{~ns}$. This may imply conformational transition within the PAZ domain itself and in the interface between the PAZ and RNase region. The RMSD for D2 simulation, after reaching equilibrium at $2 \mathrm{~ns}$, was relatively stable and

Table 1. Performed simulations and the average RMSD from initial structures calculated for $\mathrm{C} \alpha$ atoms.

\begin{tabular}{|c|c|c|c|c|c|c|}
\hline \multirow[b]{2}{*}{ Run } & \multirow[b]{2}{*}{ Structure } & \multirow[b]{2}{*}{ Method } & \multirow{2}{*}{$\begin{array}{c}\text { Simu- } \\
\text { lation } \\
\text { time } \\
{[\mathrm{ns}]}\end{array}$} & \multicolumn{3}{|c|}{$\operatorname{RMSD}[\AA ̊]$} \\
\hline & & & & all & RNase $^{1}$ & $\mathrm{PAZ}^{2}$ \\
\hline D1 & WT Dicer & GBMV & 10 & $3.8(0.8)$ & $2.4(0.3)$ & $2.9(0.8)$ \\
\hline D2 & WT Dicer & GBSW & 10 & $3.7(0.6)$ & $2.7(0.3)$ & $3.2(0.5)$ \\
\hline$\Delta \mathrm{D} 1$ & $\Delta$ Plf Dicer & GBMV & 9 & $7.1(2.6)$ & $2.7(0.3)$ & $3.3(0.7)$ \\
\hline$\Delta \mathrm{D} 2$ & $\Delta$ Plf Dicer & GBSW & 10 & $9.0(2.7)$ & $2.4(0.2)$ & $2.6(0.3)$ \\
\hline
\end{tabular}

${ }^{1}$ RNase region comprises residues 329-754,

${ }^{2} \mathrm{PAZ}$ domain comprises residues 136-250

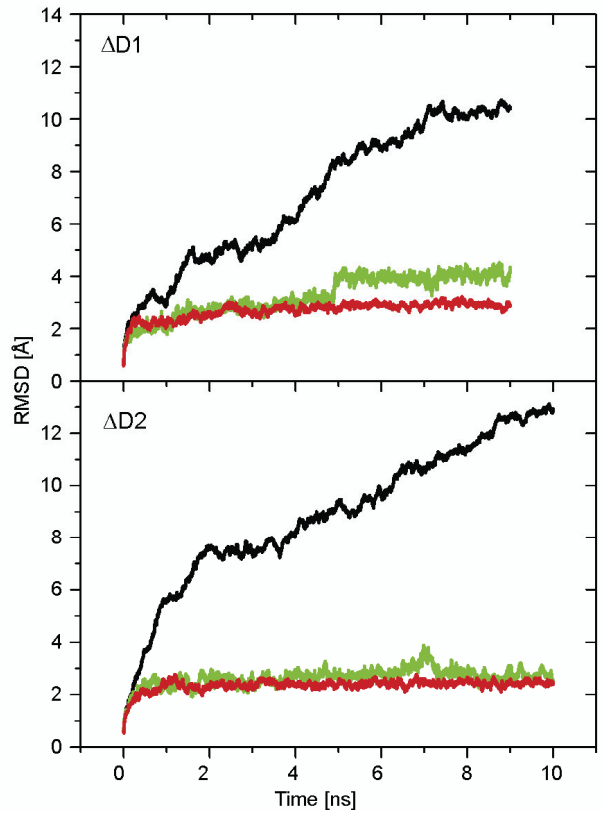

Fig. 2. Time-course of the RMSD ( $\mathrm{C} \alpha$ atoms) compared to the initial structure for the simulations presented in this work; whole molecule (black lines), PAZ domain (green lines) and RNase region (red line) 
equal to $3.9 \pm 0.3 \AA$. For simulation of $\Delta \mathrm{Plf}$ Dicer $(\Delta \mathrm{D} 1$ and $\triangle \mathrm{D} 2$ runs) the RMSD for the overall structure systematically increased, indicating that the sampling did not converge and the structure significantly departed from the initial conformation. However, the RMSD for the PAZ domain and RNase region was stable and similar as in simulations of WT Dicer. This observation indicates on the large-scale conformational change that involved the relative movement of PAZ and RNAse domains. Moreover, the RMSD between the average structures from the final $500 \mathrm{ps}$ of $\Delta \mathrm{D} 1$ and $\Delta \mathrm{D} 2$ runs was $8.7 \AA$, which shows that different conformational space was sampled in these two simulations.

\section{III.2. PCA analysis}

PCA is a mathematical technique that enables reduction of the high dimensional data to the few meaningful dimensions. This technique, in the context of MD simulations of biological macromolecules, is known as an Essential Dynamics. The essential motions, biologically relevant, can be obtained from the stable trajectories. In our simulations the essential motions can be characterized only for the WT Dicer.

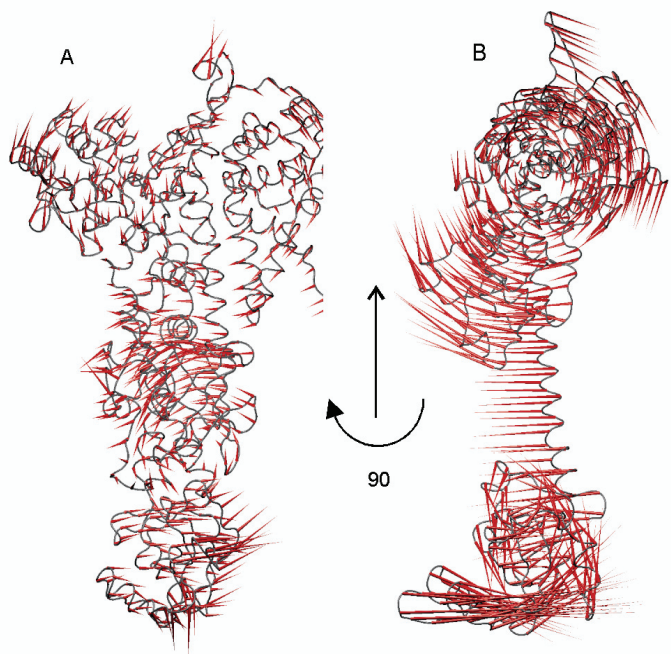

Fig. 3. Graphical representation of the first motion mode. A) WT Dicer (simulation D2). B) $\Delta$ Plf Dicer (simulation $\Delta \mathrm{D} 2$ ). Arrows show the directions and magnitudes of first eigenvector of PCA analysis for the $\mathrm{C} \alpha$ atoms. The molecules are shown in the orientation for which most of the eigenvectors are in the plane of the picture

In this work we focused on the entire conformational space sampled by the WT Dicer and $\Delta$ Plf Dicer, thus the PCA analysis was performed for the whole trajectories. For the runs of WT Dicer the first motion mode described at least $45 \%$ of total motion whereas for $\Delta$ Plf Dicer at least $81 \%$ of the total motion. The first principal component for the WT Dicer and $\Delta$ Plf Dicer from simulation D2 and $\Delta \mathrm{D} 2$, respectively, is illustrated in Fig. 3. For WT Dicer the first PCA component described the twisting motion of the
RNase IIIa and RNase IIIb around the catalytic region in the plane of the picture that is roughly parallel to the dsRNA binding plane (Fig. 3). Twisting motion about their linker region displayed also the Platform and the PAZ domain. The PAZ domain moved in the opposite direction than the Platform and the loop responsible for RNA binding in RNase IIIa. For $\Delta$ Plf Dicer the primary motion took place in the plane that is perpendicular to the plane of the primary motion of the WT Dicer.

\section{III.3. Domain motion}

To further explore the motion of the Dicer we performed a domain motion analysis with the DYNDOM

Table 2. Dynamical domains and hinge bending residues determined by DynDom program for the motion along the first eigenvector

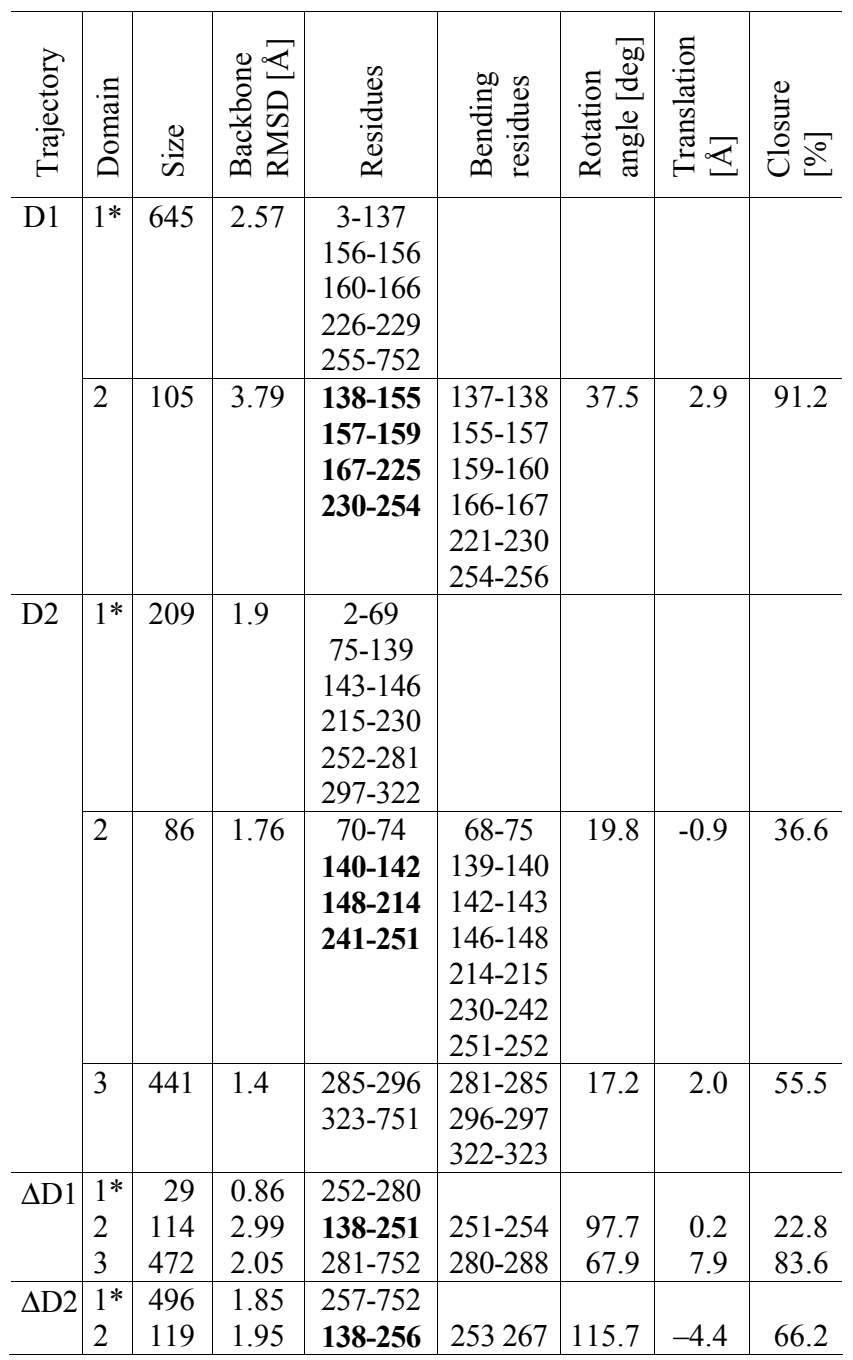

* Domain 1 is the reference conformation; the calculated angle of rotation is the angle between the second domain in the reference conformation and the second domain in the other conformation. In bold are shown the residues that belongs to the $\mathrm{PAZ}$ domain 

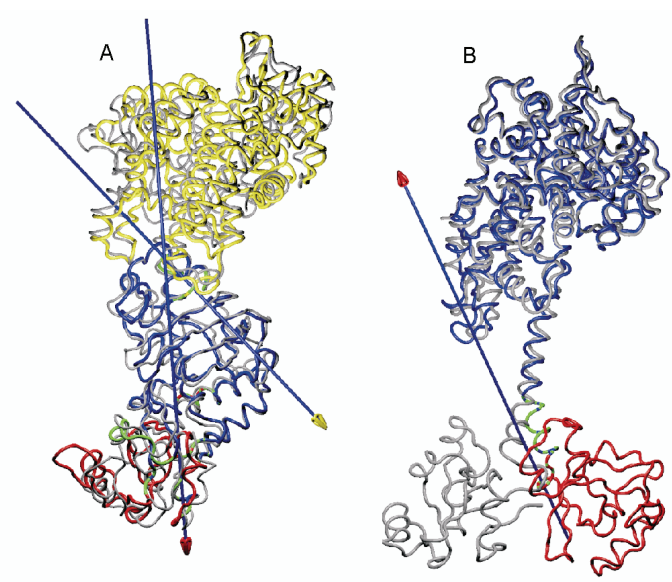

Fig. 4. Domain movement along the first eigenvector. A) WT Dicer (simulation D2). B) $\Delta$ PIf Dicer (simulation $\Delta$ D2). Fixed domain is shown in blue, the moving domain in red (and yellow for D2). The arrows indicate the hinge axis and green those regions where the hinge bending takes place

program. Both for the WT Dicer and the $\Delta$ Plf Dicer the DYNDOM identified similar dynamic domains that corresponded roughly to the structural domains (Table 2, Fig. 4). The moving domain that was detected in all simulations corresponded to the PAZ domain. The hinge for the movement of PAZ domain was formed in the loop 251-PDEFDL-256 between the PAZ and the connector helix. The PAZ in Dicer without the Platform displayed much larger movement than in the WT Dicer, showing

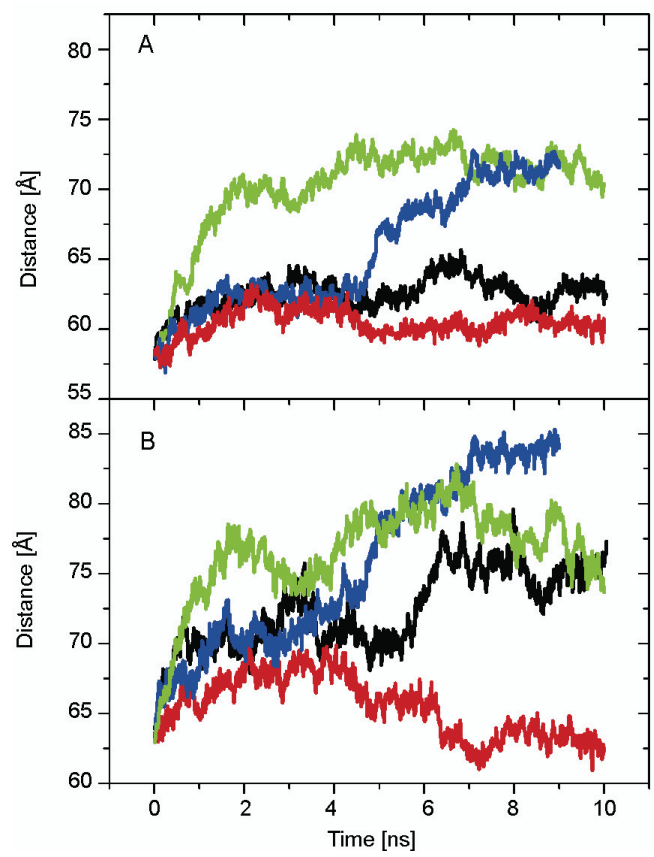

rotation angle up the $115.7^{\circ}$. In two simulations (D2 and $\Delta \mathrm{D} 1)$ the DYNDOM program identified also the second moving domain that comprised the RNase region. The hinge residues for this movement are positioned between the connector helix and the RNase IIIa region. In two remaining simulations (D1 and $\Delta \mathrm{D} 2$ ) the connector helix moved as a one domain together with the RNase region.

\section{III.4. Distances between functionally important regions}

The distance between the binding site of 3'-dinucleotide overhang and the active site in RNase IIIa determines the length of Dicer products. To examine how conformational changes of the enzyme during MD simulations influence this key distance, we have monitored distances between selected conserved $\mathrm{C} \alpha$ atoms from the RNase IIIa and PAZ domains (Fig. 5).

For simulations of the WT Dicer the distances between E336 in RNAse IIIa and L242 positioned in the $\beta$ sheet in the central part of the PAZ domain were slightly longer than in the initial structure and oscillated within $2 \AA$ of its average values. The distance from E336 to the F184 located in the appendage of the PAZ in D1 run increased and in $\mathrm{D} 2$ run decreased.

For simulations of the $\Delta$ Plf Dicer an increase of all monitored distances was observed due to departure of the PAZ domain from its initial position derived from crystal structure of the WT Dicer.

Fig. 5. The time course of the distances between the conserved residue from the catalytic region in the RNase IIIa (E336) and two selected residues from the RNA binding pocket in the PAZ domain (F184 and L242). Distances between C $\alpha$ atoms from different simulations are shown in colors: D1 - black, D2 - red, $\Delta \mathrm{D} 1$ - green, $\Delta \mathrm{D} 2$ - blue. A) The E336 - L242 distance. B) The E336 - F184 distance. C) Representation of the structure and the calculated distances 


\section{III.5. Performance of GBMV and GBSW methods}

Efficiency of the GBMV and GBSW methods was tested by running short benchmark simulations. The simulated system of the WT Dicer (756 residues) contained 11655 atoms. The simulations were run for 1000 steps with time step 1.5 fs for GBMV (1.5 ps) and 2 fs for GBSW ( 2 ps) on 1, 2, 4, 8 and 16 nodes on a computer cluster (reef.man.poznan.pl). Execution time to run the same length of simulation on one processor was about 2.4 shorter with the GBSW method than with the GBMV method. Efficiency of parallelization was slightly better for the GBSW than for the GBMV method $(0.72$ and 0.60 , for 8 processors, respectively).

\section{DISCUSSION AND CONCLUSIONS}

The role of the Dicer is to process dsRNA into the small RNA fragment of defined length in the sequence independent manner. The Dicer conformation suitable for cleavage requires appropriate positioning the RNA-binding PAZ domain in respect to the catalytic regions in RNase domains.

We have examined the flexibility of Dicer using PCA to analyze the MD trajectories generated in the implicit solvent model. In our study we have focused on the role of the Platform domain on the conformational space sampled by the Giardia Dicer. Our simulations show that the Dicer displays flexibility that can be described as a movement of two or three domains. These results obtained from the trajectories in general confirmed the previous results predicted for the whole Dicer by the NMA method that is however limited to the harmonic oscillations of the system around the energy minimum and is enabled to model multiple minima [3]. The analysis of the MD trajectories identified the PAZ domain as the main moving domain both for the Dicer with and without the Platform. In all performed simulations the hinge for the PAZ movement was found in the loop between the PAZ and connector helix whereas the NMA study suggested the hinge in P266 residue within the connector helix [3]. However, during the MD simulations of the Dicer without the Platform, the PAZ domain displayed a substantially larger movement than in the simulations of the whole Dicer. Our study shows that the Platform in Giardia Dicer clearly helps to maintain the spatial arrangement of the PAZ mainly by restricting its rotation about the axis passing the hinge region. In the simulations of Dicer without the Platform the PAZ domain displays rotations that positioned its RNA-binding pocket at the site of the protein different than the catalytic region, making the Dicer not suitable for the RNA cleavage. Since it is not clear if Dicers of higher eukaryotes, including dicer-like proteins from Arabidopsis, have a domain similar to the Platform in Giardia Dicer, it would be interesting to speculate if another domain can play a similar role as the Platform in positing the PAZ in the proper way.

On the other hand, the conformational flexibility displayed in the MD simulations of the whole Giardia Dicer is believed to allow the binding and processing of variable dsRNA substrates.

The results of simulations performed with the GBSW and GBMV methods show the same tendency although they differ in details that would also be expected within independent trajectories obtained with the same method. The simulations with the GBSW method were performed faster without the apparent compromising the quality of the results.

\section{Acknowledgements}

This work was conducted by using resources of Poznan Supercomputing and Networking Center. The authors thank G. Palik for modeling loop fragments not present in the 2FFL pdb structure using MODELLER.

Support from grant MNiSzW nr 4050/B/T02/2009/37 is acknowledged.

\section{References}

[1] I.J. MacRae, K. Zhou, F. Li, A. Repic, A.N. Brooks, W.Z. Cande, P.D. Adams, J.A. Doudna, Structural Basis for Double-Stranded RNA Processing by Dicer, Science 311,195198 (2006).

[2] I.J. MacRae, K. Zhou, J.A. Doudna, Structural Determinants of RNA Recognition and Cleavage by Dicer. Nat. Struct. Mol. Biol. 14, 934-940 (2007).

[3] I.J. MacRae, F. Li, K. Zhou, W.Z. Cande, J.A. Doudna, Structure of Dicer and Mechanistic Implications for RNAi. Cold Spring Harb. Symp. Quant. Biol. 71, 73-80 (2006).

[4] M. Dlakic, DUF283 Domain of Dicer Proteins Has a DoubleStranded RNA-Binding Fold. Bioinformatics 22, 2711-2714 (2006).

[5] H.T. Allawi, M.W. Kaiser, A.V. Onufriev, W.P. Ma, A.E. Brogaard, D.A. Case, B.P. Neri, V.I. Lyamichev, Modeling of Flap Endonuclease Interactions With DNA Substrate. Journal of Molecular Biology 328, 537-554 (2003).

[6] J. Chocholousova, M. Feig, Implicit Solvent Simulations of DNA and DNA-Protein Complexes: Agreement with Explicit Solvent VS Experiment, Journal of Physical Chemistry B 110, 17240-17251 (2006)

[7] V. Hornak, A. Okur, R.C. Rizzo, C. Simmerling, HIV-1 Protease Flaps Spontaneously Open and Reclose in Molecular Dynamics Simulations. Proceedings of the National Academy of Sciences of the United States of America 103, 915-920 (2006)

[8] M.S. Lee, F.R. Salsbury, C.L. Brooks, Novel Generalized Born Methods. Journal of Chemical Physics 116, 1060610614 (2002).

[9] M.S. Lee, M. Feig, F.R. Salsbury, Jr., C.L. Brooks, III, New Analytic Approximation to the Standard Molecular Volume Definition and Its Application to Generalized Born Calculations. J. Comput. Chem. 24, 1348-1356 (2003). 
[10] W. Im, M.S. Lee, C.L. Brooks, III, Generalized Born Model With a Simple Smoothing Function. J. Comput. Chem. 24, 1691-1702 (2003).

[11] J. Chen, W. Im, C. L. Brooks, III, Balancing Solvation and Intramolecular Interactions: Toward a Consistent Generalized Born Force Field. J. Am. Chem. Soc. 128, 3728-3736 (2006).

[12] A. Sali, T.L. Blundell, Comparative Protein Modelling by Satisfaction of Spatial Restraints, J. Mol. Biol. 234, 779-815 (1993).

[13] B.R. Brooks, R.E. Bruccoleri, B.D. Olafson, D.J. States, S. Swaminathan, M. Karplus, CHARMM: A Program for Macromolecular Energy, Minimization, and Dynamics Calculations. J. Comp. Chem. 4, 187-217 (1983).

[14] B.R. Brooks, C. L. Brooks, A. D. Mackerell, L. Nilsson, R. J Petrella, B. Roux, Y. Won, G. Archontis, C. Bartels, S. Boresch, A. Caflisch, L. Caves, Q. Cui, A.R. Dinner, M. Feig, S. Fischer, J. Gao, M. Hodoscek, W. Im, K. Kuczera, T. Lazaridis, J. Ma, V. Ovchinnikov, E. Paci, R.W. Pastor, C.B. Post, J.Z. Pu, M. Schaefer, B. Tidor, R. M. Venable, H. L. Woodcock, X. Wu, W. Yang, D.M. York, M. Karplus, CHARMM: The Biomolecular Simulation Program. Journal of Computational Chemistry 30, 1545-1614 (2009).

[15] A.D. MacKerell, D. Bashford, M. Bellott, R.L. Dunbrack, J. D. Evanseck, M.J. Field, S. Fischer, J. Gao, H. Guo, S. Ha, D. Joseph-McCarthy, L. Kuchnir, K. Kuczera, F.T. K. Lau, C. Mattos, S. Michnick, T. Ngo, D.T. Nguyen, B. Prodhom, W.E. Reiher, B. Roux, M. Schlenkrich, J.C. Smith, R. Stote, J. Straub, M. Watanabe, J. Wiorkiewicz-Kuczera, D. Yin, M. Karplus, All-Atom Empirical Potential for Molecular Modeling and Dynamics Studies of Proteins. Journal of Physical Chemistry B 102, 3586-3616 (1998).
[16] A.D. Mackerell, Jr., M. Feig, C.L. Brooks, III, Improved Treatment of the Protein Backbone in Empirical Force Fields. J. Am. Chem. Soc. 126, 698-699 (2004).

[17] M. Nina, D. Beglov, B. Roux, Atomic Radii for Continuum Electrostatics Calculations Based on Molecular Dynamics Free Energy Simulations. Journal of Physical Chemistry B 101, 5239-5248 (1997).

[18] J. Ryckaert, G. Ciccotti, H.J.C. Berendsen, Numerical Integration of the Cartesian Equations of Motion of a System With Constraints: Molecular Dynamics of N-Alkanes. J. Comp. Phys. 23, 327-341 (1977).

[19] D. Van der Spoel, E. Lindahl, B. Hess, G. Groenhof, A.E. Mark, H.J.C. Berendsen, GROMACS: Fast, Flexible, and Free. Journal of Computational Chemistry 26, 1701-1718 (2005).

[20] J. Mongan, Interactive Essential Dynamics. Journal of Computer-Aided Molecular Design 18, 433-436 (2004).

[21] W. Humphrey, A. Dalke, K. Schulten, VMD: Visual Molecular Dynamics, Journal of Molecular Graphics 14, 33-\& (1996).

[22] S. Haider, G.N. Parkinson, S. Neidle, Molecular Dynamics and Principal Components Analysis of Human Telomeric Quadruplex Multimers. Biophysical Journal 95, 296-311 (2008).

[23] S. Hayward, A. Kitao, H.J. Berendsen, Model-Free Methods of Analyzing Domain Motions in Proteins From Simulation: a Comparison of Normal Mode Analysis and Molecular Dynamics Simulation of Lysozyme. Proteins 27, 425-437 (1997).

[24] S. Hayward, H.J. Berendsen, Systematic Analysis of Domain Motions in Proteins From Conformational Change: New Results on Citrate Synthase and T4 Lysozyme. Proteins 30, 144-154 (1998).

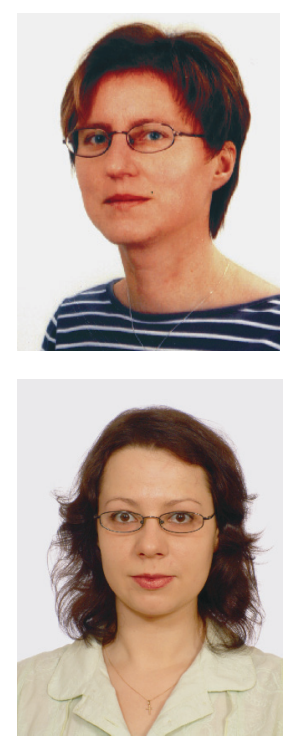

DR. Joanna SARzYnSKa received a M.S. degree in biophysics from Warsaw University and a Ph.D. from the Institute of Bioorganic Chemistry PAS, Poznan. She spent two years as a postdoctoral fellow in L. Nilsson laboratory at the Karolinska Institutet, Sweden. Using molecular dynamics and free energy perturbation method, she contributed to the correlation of experimental biochemical and thermodynamic data for RNA fragments with structural flexibility and various interaction at the atomic level. She was also a recipient of Kosciuszko Foundation Scholarship for the study of structural basis of tRNA - ribosome interactions at Georgia Institute of Technology, USA, in the laboratory of S.C. Harvey. Currently she is conducting research with Biomolecular Modelling Group at the Laboratory of Structural Chemistry of Nucleic Acids, Institute of Bioorganic Chemistry PAS, Poznan. Her research interests are focused on molecular dynamics simulations and theoretical methods to understand the effect of RNA modifications on its structure and thermodynamic stability. She is also interested in the RNA - protein interactions in the RNA interference pathway, using molecular modeling and multiscale MD simulations.

AGNIESZKa MickIEWICZ received the M.Sc. in Biotechnology in 2005 from Adam Mickiewicz University in Poznań. She is currently a Ph.D student in the Plant Molecular Biology Team, Institute of Bioorganic Chemistry, PAS.

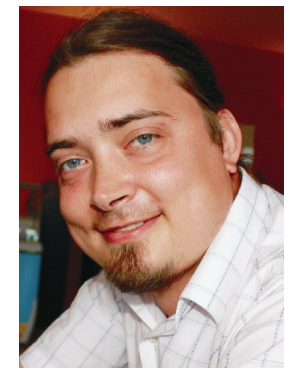

MacieJ Milostan received the M.Sc. degree in Computer Science (2002) and Ph.D. degree in technical sciences (2007) from the Poznan University of Technology. His primary field of research is bioinformatics especially protein structures prediction and analysis. However, he is also interested in security related research. Currently he is employed at the Poznan University of Technology and cooperates also with Institute of Bioorganic Chemistry PAS in Poznan and Protein Structure Prediction Center at UC Davis. 
Piotr LUKASIAK is an Assistant Professor at the Institute of Computing Science, Poznan University of Technology and at the Institute of Biochemistry, Polish Academy of Science (PAS). He was graduated from computer science department, Poznan University of Technology in 1998. He received PhD in computer science in 2004, and MBA in Carlsson School of Management, University of Minnesota in 2006. He participates as manager in many European projects (FP6) as CompuVac, MetaFunctions, Bioptrain including international Polish-French, and Polish-Italian cooperation. He worked in many countries as a visiting researcher (Rutgers University, USA; University of Minnesota, USA; INRIA, France; Davis California State University, USA), and cooperates with many other universities (e.g. UPMC - France, Hellenic Pateur Institute - Greece, Karolinska Institute - Sweden, University of Cape Town - South Africa). He received fellowship from Max-Planck Institute, Germany. In recent years he focused on bioinformatics problems working on the developing system for genetic vaccine development, protein structural and functional analysis, algorithm design, process optimalization, and management.

JACEK BłAżEwICZ born in 1951 (M.Sc. in control engineering 1974, Ph.D. and Dr. habil. in computer science in 1977 and 1980, respectively), is a professor of computer science at the Poznan University of Technology. Presently he is a deputy director of the Institute of Computing Science. His research interests

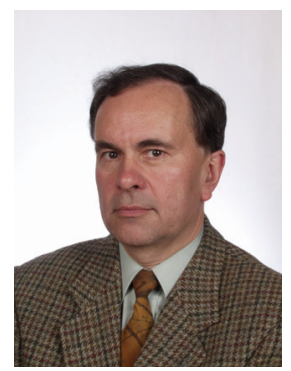
include algorithm design and complexity analysis of algorithms, especially in bioinformatics, as well as in scheduling theory. He has published widely in the above fields (over 320 papers) in many outstanding journals, among others in IEEE Trans.on Computers, IEEE Trans.on Communications, Discrete Applied Mathematics, Parallel Computing, Acta Informatica, Performance Evaluation,Journal of Computational Biology, Bioinformatics, Computer Applications in Biosciences, Operations Research, Int.Transactions of Operations Research, European Journal of Operational Research, Information Processing Letters, Operations Research Letters. He is also the author and co-author of fourteen monographs. Dr.Blazewicz is also an editor of the International Series of Handbooks in Information Systems (Springer Verlag) as well as a member of the Editorial Boards of 10 scientific journals. His science citation index exceeds 2500. In 1991 he was awarded EURO Gold Medal for his scientific achievements in the area of operations research. In 2002 he was elected for the Corresponding Member of the Polish Academy of Sciences. In 2006 he was awarded a Dr.H.C. degree from the University of Siegen.

Prof. M. Figlerowicz (MF), graduated in chemistry from the Poznań Institute of Technology in 1985. He received the $\mathrm{PhD}$ degree in biochemistry from the Adam Mickiewicz University in 1991 and the D.Sc (habilitation) degree in biology from the same university in 2000. In 2006 he acquired the title of professor of biology from the President of Poland. His research interests concern: RNA biology, especially small regulatory RNA, molecular biology of RNA viruses and retroviruses, functional genomics. He has been the principal investigator of numerous scientific projects sponsored by the Polish Ministry of Science and High Education, European Union and UNESCO. He is the author or co-author of 91 articles and 122 published scientific communications. As an invited speaker, he has given 47 lectures and seminars. Currently, he is the head of the Laboratory of Plant Molecular Biology at the Institute of Bioorganic Chemistry (ICHB) Polish Academy of Sciences, Poznan, the head of the Center of Excellence for Nucleic Acid Based Technologies (CENAT), located at the ICHB and the head of Laboratory of Molecular Virology at ICHB. He is also a member of several scientific boards, the editorial board of Journal of RNAi and Gene Silencing, Oxford United Kingdom and a member of the editorial board of Biotechnologia - Journal of the Biotechnology Committee of the Polish Academy of Sciences.

TAdeusz KUlińSKI, PhD, DSci. is a head of Biomolecular Modelling Group at the Institute of Bioorganic Chemistry, Polish Academy of Sciences, Poznań, Poland. He graduated in physics and received the PhD degree in physics from the Adam Mickiewicz University. In1984 he joined the Institute of Bioorganic Chemistry, Polish Academy of Sciences, where he received the D.Sci. (habilitation) degree in molecular biology. His current major interests are structural studies of RNA and proteins in order to understand the relationship between the structures of these molecules and their biological functions and molecular recognition. He is using different computational approaches for modeling of nucleic acids and proteins structure and interactions, determination of non-standard hydrogen bonding, water mediated hydrogen bonding and their participation in the stabilization of RNA structural domains (various regions of ribosomal RNA, retroviral RNA's). He is also working on the optimization of different numerical methods to estimate free energy, or methods combining molecular mechanics and continuum models for the prediction of binding affinities and stability changes of RNA, for the prediction of the properties of new RNA analogs in aqueous solution as well as new compounds as drug candidates. 